\section{IMPAIRED CONSCIOUSNESS DUE TO INJURY OF ASCENDING RETICULAR ACTIVATING SYSTEM}

Keywords

consciousness • ascending reticular activating system • caudate nucleus • diffusion tensor tractography
A 75-year-old female patient presented with impaired consciousness, and was diagnosed with multiple cerebral infarctions in both hemispheres, including bilateral caudate nuclei and right cingulums (Fig.1-A). Although she was diagnosed with a left pontine infarct eight months prior, she recovered fully without any sequelae. At one month after the multiple infarcts, she was transferred to the department of rehabilitation and revealed severely impaired consciousness, with a Glasgow Coma Scale score of 4 (eye opening: 2 , best verbal response: 1 , and best motor response: 1) and Coma Recovery Scale-Revised score of 3 (auditory

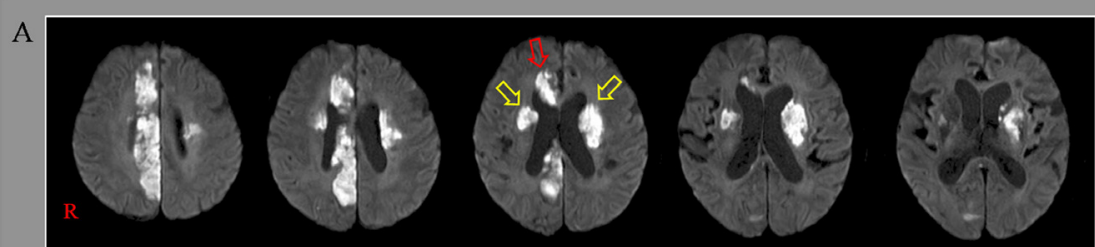

$\mathrm{B}$

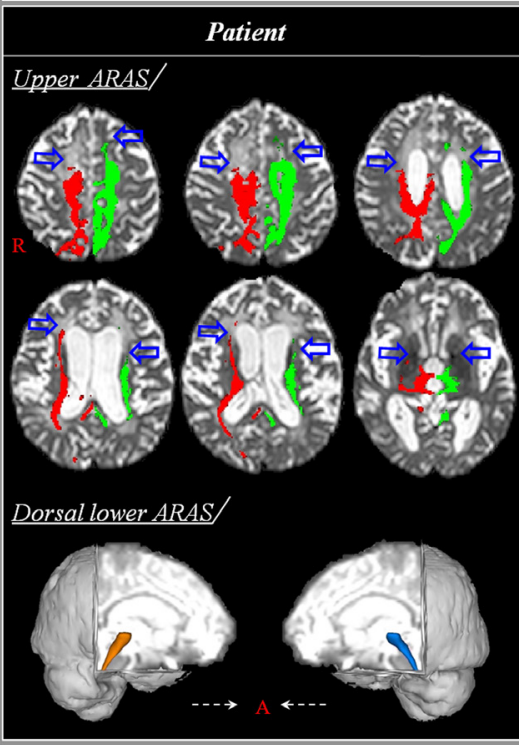

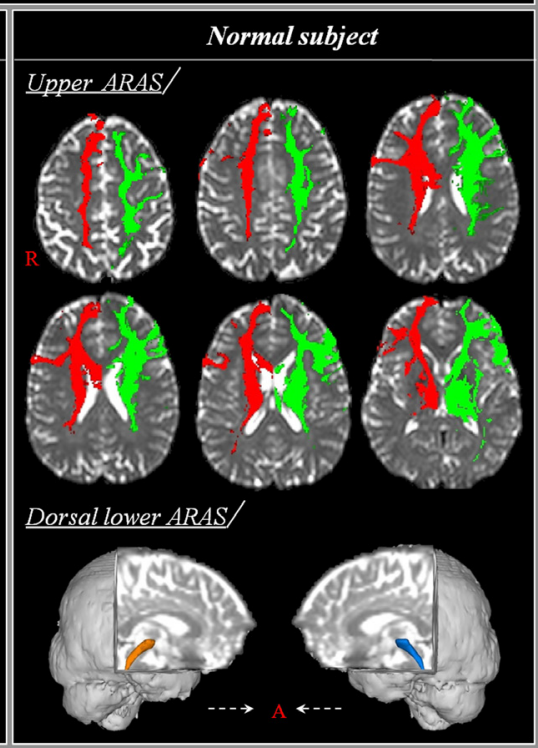

Fig. 1. (A) Brain MR images at onset show multiple cerebral infarctions in both hemispheres including bilateral caudate nuclei (yellow arrows) and right cingulum (red arrow). (B) Results of diffusion tensor tractography for the ascending reticular activating system (ARAS). On 3-month diffusion tensor tractography, the neural connectivity in the upper ARAS between the thalamic intralaminar nucleus and the cerebral cortex is decreased in both prefrontal cortices and right cingulate cortex (blue arrows). By contrast, the lower ARAS between the pontine reticular formation and the thalamic intralaminar nucleus does not show significant abnormality compared with a normal subject (72 year-old female).
Sung Ho Jang',

Jun Lee',

Hyeok Gyu Kwon ${ }^{3 *}$

'Department of Physical Medicine and Rehabilitation, College of Medicine, Yeungnam University

${ }^{2}$ Department of Neurology, College of Medicine, Yeungnam University

${ }^{3}$ Department of Physical Therapy, College of Health Sciences, Catholic University of Pusan, 57 Oryundae-ro, Geumjeong-gu, Pusan, 46252, Republic of Korea

Received 06 December 2018 accepted 17 December 2018

function: 0 , visual function: 1 , motor function: 1 , verbal function: 0 , communication: 0 , and arousal: 1) [1,2]. She underwent comprehensive rehabilitation for two months, although her consciousness did not recover significantly, with a Glasgow Coma Scale score of 8 (eye opening: 4 , best verbal response: 1 , and best motor response: 3 ) and Coma Recovery ScaleRevised score of 8 (auditory function: 1, visual function: 3 , motor function: 2 , verbal function: 0 , communication: 0 , and arousal: 2) [1,2].

Diffusion tensor imaging data was acquired at three months after onset of head trauma using a 1.5T with 32 gradients. Imaging parameters were as follows: acquisition matrix $=96 \times 96$; reconstructed to matrix $=192 \times 192$; field of view $=240 \times 240 \mathrm{~mm}^{2}$; repetition time $=10,398 \mathrm{~ms}$; echo time $=72 \mathrm{~ms} ; \mathrm{b}=1000 \mathrm{~s} /$ $\mathrm{mm}^{2}$; and a slice thickness of $2.5 \mathrm{~mm}$. For the connectivity of the upper ascending reticular activating system (ARAS), thalamic intralaminar nucleus (ILN) was used as a seed region of interest (ROI) at the level of the commissural plane [3]. For reconstruction of the lower ARAS, a seed ROI was placed on the reticular formation at the mid-pons and a target ROI was placed on the thalamic ILN at the level of the commissural plane [4]. The thresholds of 10 streamlines for the upper ARAS and 2 
streamlines for the lower ARAS were applied for the results of fiber tracking.

On 3-month diffusion tensor tractography (DTT), the neural connectivity in the upper ARAS between the thalamic ILN and the cerebral cortex was decreased in both prefrontal cortices and right cingulate cortex (Fig.1-B). By contrast, the lower ARAS between the pontine reticular formation and the thalamic ILN did not show significant abnormality.
In conclusion, injury of the upper ARAS between the thalamic ILN and the cerebral cortex following multiple cerebral infarct in both hemispheres was demonstrated in a stroke patient. Our results suggest that evaluation of the ARAS using DTT would be necessary to understand the state of the ARAS in stroke patients with impaired consciousness following multiple pathologies.

\section{Conflict of interest}

The authors declare that they have no conflict of interest.

\section{Acknowledgements}

This work was supported by the National Research Foundation of Korea (NRF) grant funded by the Korean Government(MSIP) (No. 2018R1A2B6000996).

\section{References}

[1] Teasdale G., Jennett B., Assessment of coma and impaired consciousness. A practical scale, Lancet, 1974, 2, 81-84

[2] Giacino J.T., Kalmar K., Whyte J., The JFK Coma Recovery ScaleRevised: measurement characteristics and diagnostic utility, Arch Phys Med Rehabil, 2004, 85, 2020-2029

[3] Jang S.H., Lim H.W., Yeo S.S., The neural connectivity of the intralaminar thalamic nuclei in the human brain: a diffusion tensor tractography study, Neurosci Lett, 2014, 579, 140-144
[4] Yeo S.S., Chang P.H., Jang S.H., The ascending reticular activating system from pontine reticular formation to the thalamus in the human brain, Front Hum Neurosci, 2013, 7, 416 